\title{
Assessment of Periodontal Status of Surgically Exposed and Orthodontically Aligned Impacted Maxillary Canines
}

\author{
Coșarcă Adina¹, Păcurar Mariana², Petrovan Cecilia1', Ormenișan Alina1 \\ Department of Oral and Maxillofacial Surgery, Faculty of Dental Medicine, University of Medicine and Pharmacy, Tîrgu Mureș, Romania \\ 2 Department of Pedodontics and Orthodontics, Faculty of Dental Medicine, University of Medicine and Pharmacy, Tîrgu Mureș, Romania
}

\begin{abstract}
Aim: The aim of this study was to compare the periodontal status of impacted canines after 5 years following completion of the combined surgical and orthodontic treatment.

Materials and methods: We examined 20 labially impacted canines and 20 palatally impacted canines at 5 years after the end of treatment. We assessed the periodontal status of these teeth.

Results: Different outcomes were found regarding the probing depth and the amount of keratinized gingiva in the two mentioned groups of teeth. Conclusions: The assessed periodontal indices may signal the appearance of a periodontal disease around the teeth that were surgically and orthodontically treated.
\end{abstract}

Keywords: impacted canine, periodontal, surgical-orthodontic treatment

Received: 14 July 2013 / Accepted: 8 January 2014

\section{Introduction}

It is known that maxillary canines remain impacted more often than the mandibular canines, and the inclusion can be buccal or palatal [1]. The treatment focuses mainly on the exposure and on the orthodontic realignment of the impacted tooth. There are situations when canines erupt spontaneously after their surgical discovery.

The surgical exposure techniques may affect the level of keratinized gingiva attached to the causal tooth and the periodontal health status of the treated tooth. The surgical therapy can be completed by a gingivectomy, an apically positioned flap technique or by a tunnel technique [2].

After completing the orthodontic treatment the patient must be monitored by regular follow-up visits, which would focus on maintaining the orthodontic results and also on assessing periodontal health of the teeth, especially of those disimpacted teeth where the sacrifice of gingival and osseous tissue was important during the treatment. Regardless of the initial impacted position, at the end of treatment canine impaction encompasses mostly aesthetic problems, as the inclusion is located in the area of the dental arch where aesthetics prevail. In this region the width of attached gingival and also the amount of gingival recession is extremely important.

\section{Objectives}

The aim of this study was to assess some simple periodontal parameters in the disimpacted maxillary canines after 5 years from the end of the combined surgical-orthodontic treatment.

\section{Materials and methods}

The study included two groups of patients: the first one consisted of 20 patients with initially buccally impacted maxillary canines that were surgically exposed and orthodontically realigned on the dental arch, and the second group represented by 20 patients with surgically and orthodontically recovered palatally impacted upper canines. The patients were aged between 18 and 25 years.

The inclusion criteria during this study were as follows: the existence of the bucally or palatally impacted canine, completed surgical and orthodontic therapy (at least 5 years after the end of the treatment), the alignment of canine on the dental arch, using a surgical exposure technique which involved the excision of mucosa over the impacted tooth (gingivectomy), and patient without any associated diseases. No account was taken of the type of braces worn by the patients, the duration of treatment, the brushing method applied by each patient, gender or the depth of dental impaction.

All patients enrolled in the study were assessed by a single physician (author of the paper), using visual examination and palpation with a graduated periodontal probe. The periodontal probe was marked at every millimeter from 1 to $10 \mathrm{~mm}$. Gingival index, plaque index, probing depth and gingival recession were assessed.

Gingival index was recorded by Silness and Löe method and it was based on the following criteria: $0=$ normal gingiva; 1 = mild inflammation with slight change in color and slight edema but no bleeding on probing; 2 = moderate inflammation, redness, edema, glazing and bleeding on probing; 3 = severe inflammation, characterized by marked redness and gingival edema, ulceration with tendency to spontaneous bleeding. 
Plaque index was assessed using the Silness and Löe plaque index with the following scores: $0=$ no plaque; $1=\mathrm{a}$ film of plaque adhering to the free gingival margin and adjacent area of the tooth and it may be observed in situ only after application of a disclosing solution or by using a probe on the tooth surface; 2 = moderate accumulation of soft deposits within gingival pocket, or on the tooth and gingival margin, that can be seen with the naked eye; 3 = an abundance of soft matter within the gingival pocket and/or on the tooth and gingival margin, in all these areas. At each tooth all four dental surfaces $(\mathrm{M}, \mathrm{D}, \mathrm{B}, \mathrm{P})$ were examined and the average plaque index was also calculated for each canine.

The probing depth was assessed using a periodontal probe and six specific sites were selected on each tooth: distofacial, facial, mesiofacial, disto-palatal, palatal, and mesiopalatal surface. The deepest reading per tooth was recorded.

Gingival recession was appreciated measuring the distance between the cement-enamel junction (CEJ) and the free gingival margin.

Subsequently the recorded values were compared between the two groups of realigned canines.

Data were processed using Microsoft Excel and Graphpad Prism 5 software. Mann-Whitney non-parametric test was used for unpaired data and the threshold of significance was set to $\mathrm{p}=0.05$.

\section{Results}

Gingival index highlighted severe inflammation and spontaneous bleeding in two patients of the first group. In the other patients the gingival index had values mostly of 1 and 2 .

The average gingival index of the first group was 1.500, with a standard deviation of 0.8885 and a standard error of 0.1987 . In the second group the average value was 0.7500 , with a standard deviation of 0.7164 and a standard error of 0.1602 . The comparison of the two groups has lead to statistically significant results $(\mathrm{p}<0.05)$ (Table I).

Regarding the plaque index, only one patient presented the maximal score of 3 and the rest of the enrolled patients had predominantly the score of 1 .
Table I. Comparison of the two groups

\begin{tabular}{lccc}
\hline Parameter & Group I & Group II & p value \\
\hline Gingival index & 1.500 & 0.750 & 0.0080 \\
Plaque index & 1.000 & 0.750 & 0.2458 \\
Probing depth & 0.850 & 1.450 & 0.0040 \\
Gingival recession & 1.600 & 0.500 & 0.0003 \\
\hline
\end{tabular}

The average plaque index of the first group was 1.000, with a standard deviation of 0.6489 , and standard error of 0.1451 . The second group showed an average plaque index of 0.7500 , with a standard deviation of 0.7864 , and a standard error of 0.1758 . The comparison between the two groups has lead to differences that were statistically not significant $(\mathrm{p}>0.05)$.

The average probing depth of the first group of patients was 0.8500 , with a standard deviation of 0.6708 and a standard error of 1.500 . In the second group the average value of the probing depth index was 1.450 , with a standard deviation of 0.6863 and a standard error of 0.1535 . The differences between the two groups were statistically significant $(\mathrm{p}<0.05)$ (Figure 1, Table I).

Regarding the gingival recession index the average value in the first group was 1.600, with a standard deviation of 1.046 and a standard error of 0.2340 . In the second group the average value was 0.5000 , with a standard deviation of 0.5130 , and a standard error of 0.1147 . Statistically significant differences were found between the values of the two studied groups, $\mathrm{p}=0.0003$ (Figure 2).

\section{Discussion}

The aforementioned indices for assessing the periodontal health and hygiene status can be applied easily, quickly and repeatedly. The readings can be recorded in the medical file of the patient and have medical, scientific and judicial role $[3,4]$.

The gingival index demonstrates the severity of gingival inflammation around the studied teeth.

Although differences between the two groups were statistically significant, the overall gingival inflammation

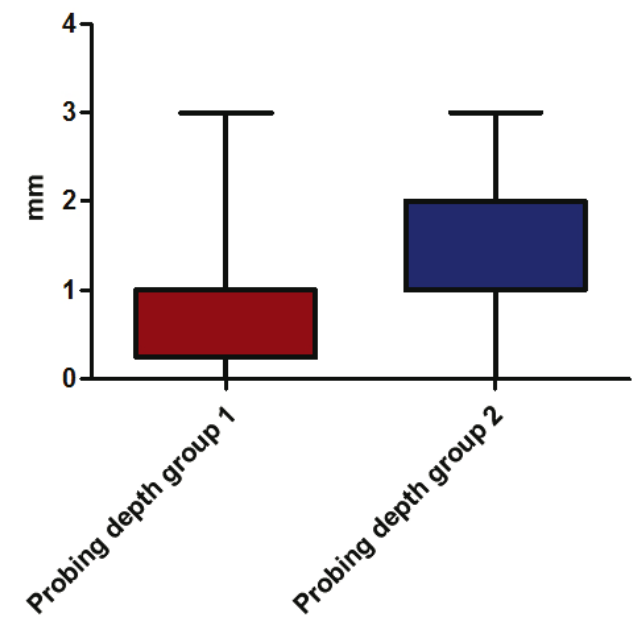

Fig. 1. The average probing depth

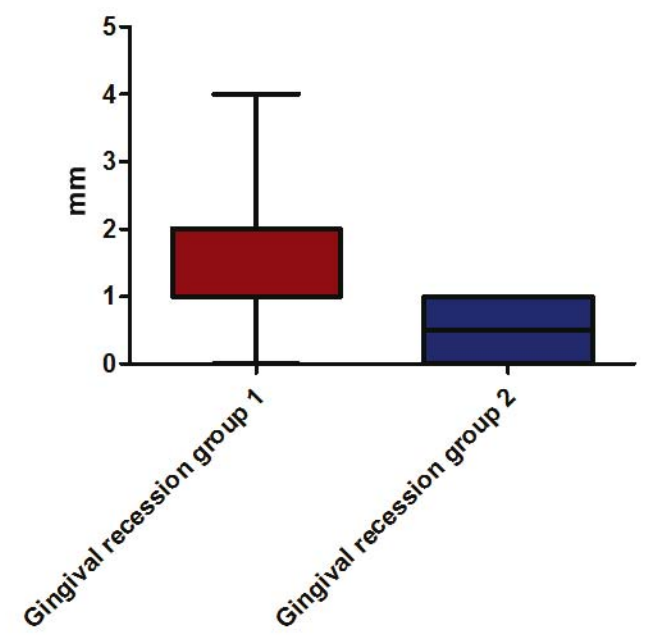

Fig. 2. The average gingival recession index 


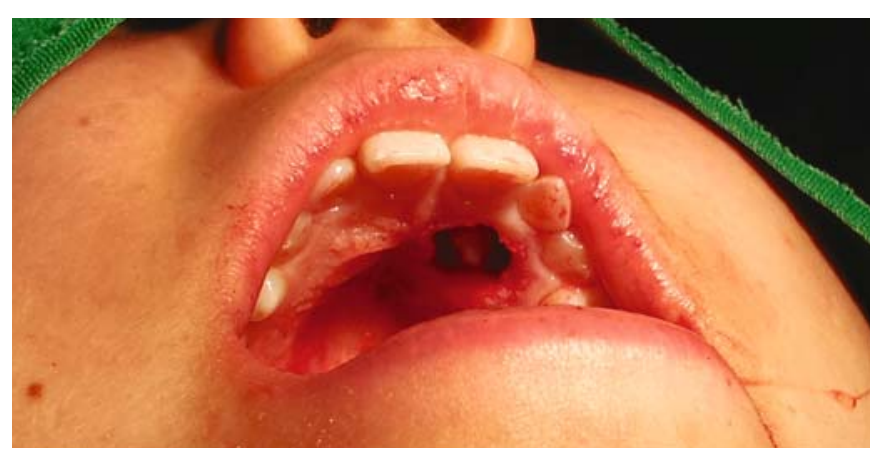

Fig. 3. Palatal inclusion (gingivectomy)

around the buccally impacted and realigned canines was very severe, two of these patients exhibiting an average gingival index of 3 , with advanced inflammation, congestion, stasis and spontaneous bleeding.

At the assessment of plaque index the prevailing values were 1 and 2 in both groups, thus plaque accumulation of the enrolled patients was within the normal limits up to quite satisfactory. Another study that compared palatally impacted and surgical-orthodontic realigned canines with normally erupted canines had highlighted statistically significant differences between the two groups of teeth, with higher plaque scored on the included and subsequently treated canines [5].

To evaluate the accuracy of a completed surgical and orthodontic treatment of the impacted teeth the level of the attached gingival and the probing depth should be appreciated, which are actually the parameters for assessing the success of such therapies.

Using surgical techniques that remove the gingival tissue located over the impacted tooth is detrimental for the keratinized gingival, especially in the buccal area, as it was demonstrated by the present study, where the gingival recession was more pronounced. When using this technique healing occurs by secondary granulation and it requires a longer period of regeneration in comparison with other techniques which imply flaps and surgical sutures. The unsutured wounds require a period of at least 3-4 weeks of healing, and the normal structure of the mucosa can be assessed only after 6 months [6]. Meanwhile the interference of harmful intraoral factors, such as poor oral hygiene, may affect the slow and difficult healing process and thus can lead to gingival inflammation. The combination with an incorrect dental brushing technique can accelerate the gingival recession. If removal of the osseous substrate and keratinized gingival was substantial during the surgical exposure, this loss would have a great impact later on the periodontal health status of the realigned tooth.

The difference between the probing depth values of the two groups could be explained by the destruction of the osseous substrate more severe on the palatal aspect due to the difficult access to the impacted tooth at the moment of surgical exposure or due to an incorrect direction of the tooth to the new location within the dental arch (Figure 1). The study did not take into account the intraosseous depth of the impacted teeth, which may also be related to the depth of periodontal probing after realigning the teeth. Although the results are statistically significant, the values obtained at periodontal probing after completing the therapy were predominantly varying between 1 and 2 millimeters, which represent the physiologic depth of the sulcus gingivalis [7].

Minimal gingival retraction (the level of attached gingiva) is considered the goal of an adequate surgical and orthodontic treatment, as the keratinized gingival has an important aesthetic role and in the composition of the smile [8].

A study conducted in 2007 which compared normally erupted canines with impacted canines treated surgical and orthodontic with a technique that minimized the destruction of the attached gingiva did not show any differences between the two groups of teeth in terms of probing depth and level of attached gingival [9].

The results of the present study demonstrate that using gingivectomy as surgical exposure technique can affect the aesthetics at the end of treatment. Currently this surgical technique is used only exceptionally when dental impaction is coronal to the mucogingival junction. The latest surgical exposure and orthodontic traction techniques use a minimal osseous distruction by creating a tunnel similar to that of the physiological eruption process or by the apical positioning of the flap of mucosa that covers the impacted tooth $[2,8]$.

\section{Conclusions}

1. Buccal dental impaction generates most problems for the surgeon because of the attached gingival tissue in this area with an important aesthetic role, which appeals to preservation during realignment.

2. Using gingivectomy as surgical exposure technique should be carefully evaluated in the preoperative stage, considering its aesthetic complications that may follow after the surgical and orthodontic treatment.

3. The patients should be aware and informed that the treatment of dental impaction and the gained results should be monitored also after completing the therapy, through regular control and follow-up visits.

4. Considering that during the surgical and orthodontic procedures the patient wears very retentive orthodontic braces, he or she should be instructed on the most suitable dental brushing technique and also on using auxiliary oral hygiene devices, to avoid an eventual gingival inflammation due to plaque accumulation.

5. Probing depth and gingival recession should be monitored throughout the surgical and orthodontic treatment and also after completing the therapy, as these parameters may be essential signs of a periodontal disease in the area of the treated teeth. The annual assessment could diagnose earlier the onset of the periodontal disease in all teeth that underwent surgical and orthodontic treatment. 


\section{References}

1. Bucur A. Compendiu de chirurgie oro-maxilo-facială. Q Med Publishing 2009, vol.l,115-116;

2. Kokich VG. Surgical and Orthodontic Management of Impacted Maxillary Canines. Am J Orthod Dentofacial Orthop. 2004;126:278-83.

3. Dumitriu H. Parodontologie. Editura Viata Medicala Romaneasca 2009,163-288.

4. Monea A, Monea M. Parodontologie. Editura Ardealul, 1999, 27-32.

5. Hansson C, Rindler A. Periodontal conditions following surgical and orthodontic treatment of palatally impacted maxillary canines - a followup study. The Angle Orthodontist. 1998;68(2):167-172.
6. Burlibașa C. Chirurgie orală și maxilo facială. Editura Medicală. 2001, 259263

7. Lindhe J, Thorkild K, Niklaus P. Clinical Periodontology and Implant Dentistry, Fourth Edition, Blachwell Munksgaard Publishing Company, 2003, 3-14.

8. Crescini A, Nieri M, Buti J, Baccetti T, Pini Prato G. Orthodontic and Periodontal Outcomes of Treated Impacted Maxillary Canines. Angle Orthodontist. 2007;77(4):571-577.

9. Crescini A, Buti J, Nieri M, et al. Short- and long-term periodontal evaluation of impacted canines treated with a closed surgical-orthodontic approach. J Clin Periodontol. 2007;34(30):232-42. 